\title{
PENSAR GEOGRÁFICAMENTE: UNA APROXIMACIÓN A LA ENSEÑANZA DE LA GEOGRAFÍA DESDE EL CURRÍCULUM AL AULA
}

\author{
Francisca Armijo Baeza ${ }^{1}$
}

\begin{abstract}
RESUMEN/ ABSTRACT
El siguiente artículo tiene por objetivo analizar las orientaciones curriculares en la enseñanza de la Geografía en octavo básico y evaluar su impacto en el proceso de enseñanza-aprendizaje en la asignatura de Historia, Geografía y Ciencias Sociales. Este análisis se realiza a partir de un estudio que asumió una metodología de investigación cualitativa cuya base fue la revisión de las Bases Curriculares (código curricular) de 2013 y el Programa de Estudio (currículum) de octavo básico, elaborados por el Ministerio de Educación de Chile, además de la revisión de material bibliográfico sobre la enseñanza del conocimiento geográfico en el contexto escolar, desde la perspectiva de la Geografía Crítica, la que permitió sintetizar las principales conclusiones. Finalmente, a partir de la investigación, el artículo concluye que, a pesar del discurso instalado en las Bases Curriculares de 2013 sobre la relevancia del pensamiento geográfico dentro del currículum y programa de estudio, la Geografía no ha sido incorporada como una disciplina que dialogue con la Historia y las Ciencias Sociales y permita a nuestros estudiantes pensar geográficamente. Hasta ahora, la Geografía sigue siendo considerada, como lo fue en el siglo XX, una ciencia auxiliar y depositaria del ideal de Estado nación.
\end{abstract}

Palabras clave: bases curriculares; orientación curricular; pensamiento geográfico; octavo básico; aprendizaje significativo.

The following article aims to analyze the curricular orientations on teaching of Geography in the eighth grade and assess its impact on teaching-learning History, Geography and Social Sciences. For this purpose, the study assumed a qualitative research methodology based on the bibliographic review of the Curricular Bases of 2013 and the Eighth Grade's Study Program for History, Geography and Social Sciences areas, prepared by the Ministry of Education of Chile. This study also used a bibliographic review about the teaching of geographycal knowledge at school from the perspective of the critical Geography. That allows us to summarize the main results. Based on this reaserch, the article concludes that despite the installed discourse on the Curricular Bases in 2013 about the relevance of geographic thinking, Geography has not been incorporated as a discipline that dialogue with History and Social Sciences. This practice does not allow the students to think

\footnotetext{
${ }^{1}$ Docente de Historia y Geografía, Colegio Santa Marta, Chile; francisca.armijo.baeza@gmail.com.
} 
geographically either. Until now, Geography follows the same path as in the twentieth century, like a peripheral science and as a holder of the nation-state ideal.

Keywords: curricular bases; curricular orientation; geographic thinking; eighth grade; significant learning.

\section{INTRODUCCIÓN}

Si contemplamos un trigal o un maizal, somos conscientes de que los surcos, las sementeras, las barreras de los campos, de seto o alambrada, indican relaciones de producción y de propiedad (...). Así, la pertenencia de un espacio a la naturaleza le dispensa de entrar en el universo de las relaciones sociales de producción. Pero el carácter de estos espacios en los que predominan las peculiaridades naturales o que contienen objetos dotados de dichas particularidades declina progresivamente. Y con él, se aleja la Naturaleza.

Henri Lefebvre, 1974, p.139.

A pesar de estar declarados los énfasis de la asignatura de Historia, Geografía y Ciencias Sociales en las Bases Curriculares (perspectiva multidisciplinar, pensamiento histórico, pensamiento geográfico, etc.), en el proceso de enseñanza y evaluación que se materializa en el Programa de Estudio existe una tendencia hacia el pensamiento temporal. Asimismo, en este último, los énfasis de las Bases aparecen fragmentados y orientados al cumplimiento de indicadores de evaluación secuenciados clase a clase que no incorporan las herramientas ni aprendizajes propios de las disciplinas consideradas, en particular, de la Geografía. Del mismo modo, la adecuación de los contenidos al contexto de la escuela y de los estudiantes depende muchas veces de la creatividad curricular que pueda desarrollar el docente, en tanto asunción de riesgos (Magendzo, 2008, p.181). En este sentido, si bien existe una flexibilidad sobre el cómo abordar los contenidos, predomina una tendencia obvia a replicar las orientaciones curriculares de la asignatura, puesto que "la mayoría del profesorado tiene poco tiempo para debatir y pensar cómo llegan hasta las puertas de sus 
aulas los contenidos educativos" (Souto, 2011, p.30), lo que se traduce en la reproducción de las actividades sugeridas en los programas, cuestión que, como se analizará, afecta el trabajo multidisciplinar.

A partir de lo anterior, este estudio se posiciona desde la perspectiva de la Geografía Crítica, en particular desde los aportes de Milton Santos y Xosé Souto, quienes han planteado la necesidad de que la Geografía colabore de forma directa en el análisis de características y factores que influyen en el aprendizaje de los estudiantes como "sus deseos, expectativas sociales, valores culturales dominantes, relaciones de sus viviendas con el sistema urbano dominante o las relaciones sociales y familiares que determinan las emociones, afectos y razonamientos del alumnado" (Souto, 2011, p.31).

\section{CURRÍCULUM Y CÓDIGO CURRICULAR}

Antes de abordar los objetivos del estudio es necesario aclarar conceptualmente qué se entenderá por currículum y código curricular, ya que a lo largo del artículo dialogan dos fuentes bibliográficas - las Bases Curriculares y el Programa de Estudio para octavo básicorelativas a ambos conceptos.

Por currículum, se entenderá la selección del conocimiento y destrezas que deberán ser transmitidas por la educación, la "selección de contenidos y fines para la reproducción social” (Lundgren, 1992, p.20). De esta forma, como plantea Lundgren, el currículum se relacionará con la definición de métodos y esquemas de cómo deben desempeñarse y materializarse los contenidos seleccionados. Por otra parte, lo que define esta selección y organización de qué es lo que debería enseñarse, es la fundamentación de que el currículum

puede convertirse en un producto, como dice el autor, en un suceso en el proceso de enseñanza y aprendizaje. En una representación.

A su vez, por código curricular se entenderá "el conjunto de principios sobre cómo debe seleccionarse, organizarse y transmitirse el conocimiento y destrezas" (Lundgren, 1992, p.22). El código curricular es un texto producido para la enseñanza y que estructura diversos campos del conocimiento que tienen como finalidad la reproducción de un 
determinado modelo de sociedad, y que dan sustento al desarrollo teórico e ideológico de un ideal educativo. En esta línea, los códigos curriculares expresan distintos puntos de vista y perspectivas sobre los propósitos de la educación.

De esta forma, el currículum corresponde al Programa de Estudio de octavo básico, ya que desglosa los objetivos de aprendizaje, las habilidades y los indicadores de evaluación; y el código curricular corresponde a las Bases Curriculares, ya que presenta los énfasis curriculares y la perspectiva educativa que asume el Programa de Estudio.

Dicho lo anterior, es preciso añadir que, como señala Grundy (1998), el currículum debe ser entendido como una construcción cultural, como "un conjunto de prácticas educativas humanas” (p.20). En este sentido, es relevante señalar que no es posible comprender el desarrollo del currículum sin reconocer que el proceso de su creación está mediado y tiene como antecedente la experiencia humana relativa a los procesos educativos. Tanto profesores como estudiantes a lo largo de su vida se han comprometido, consciente o inconscientemente, con prácticas curriculares (Grundy, 1998). El carácter de la construcción del currículum de las escuelas constituye, por tanto, parte integrante de su cultura, de las circunstancias y contextos que han marcado el desarrollo social, político y económico de las sociedades. Según Grundy, no es posible entender al currículum como “algo aparte" o fuera de la interacción humana. Es por ello que en la problematización del currículum es necesario reconocer los intereses que han permeado el proceso de constitución de las realidades educativas, pero también las concepciones que han definido el "qué hacer" de las escuelas y de las instituciones educativas.

Esto último ha sido desarrollado por Carr (2005) quien plantea que ambos, currículum y código curricular, son nociones fundamentalmente normativas. Por esta razón, resulta importante replantearse las prácticas curriculares en la escuela y la forma en que se construye el conocimiento, como dinámicas que no están fuera de las personas, sino que son elaboradas por ellas en conjunto, tanto en su significación, como en sus expresiones, su comprensión y su valoración. De esta forma, el currículum se inserta y dinamiza en la escuela, donde se produce la interacción entre actores que se comunican y "se hacen en términos de sus propios códigos culturales" (Pinto, 2008, p.103). 
Aclarada la perspectiva conceptual que se adopta en este artículo, a continuación se presenta una breve contextualización de lo que ha sido la enseñanza de la Geografía en Chile, para luego profundizar en el tratamiento pedagógico-curricular que ha tenido la disciplina geográfica y sus consecuencias.

\section{LA ENSEÑANZA DE LA GEOGRAFÍA EN CHILE}

Desde los inicios de la educación formal en el país, la Geografía ha estado presente en los planes escolares con variaciones relativas a la construcción del Estado. Desde que el Estado chileno reconoce su responsabilidad en el desarrollo de la educación nacional, en la segunda mitad el siglo XIX y aún con un acceso limitado a la educación (Núñez, 1997, párr. 4), la enseñanza de la Geografía estuvo muy relacionada con las áreas de la Matemática y la Física, desde una perspectiva autónoma que ha sido catalogada como precientífica o premoderna. En este período, la Geografía propuesta en los planes de estudio fue fiel reflejo de la Geografía académica que se abocaba a la tarea de describir la superficie terrestre - por ello los contenidos asociados eran lo que se ha conocido como la Geografía General. Como materiales de estudio se utilizaban textos traídos desde Europa, mapas del mundo y globos terráqueos. Así, la asignatura de Geografía contenía lecciones de cosmografía, Geografía General y Política, descripción de los continentes y tierras polares. En cuanto a la Geografía Política se dedicaba especial atención a la división política de la Tierra, donde Europa ocupaba un lugar privilegiado de descripción, dejando de lado, o fragmentados, a Asia, África e inclusive América.

Este panorama cambia a comienzos del siglo XX, contexto en el que la figura de la república comenzaba a consolidarse, ya que la Geografía se orienta hacia un cariz nacionalista y subsidiario de la Historia (Miranda, 2012), para reforzar la instalación de una identidad nacional y un ideal patrio chileno. Esta Geografía, de corte positivista, y donde los contenidos se definieron como extensas y detalladas descripciones del territorio nacional, se mantuvo en Chile desde el plan de estudios concéntrico de 1889, con pequeños ajustes pero sin modificaciones profundas, hasta la reforma curricular de 1996. La Geografía Regional de Chile, la Geografía Regional del Mundo, y los Hitos de la Geografía 
Local, Regional y Nacional marcaron la orientación de la disciplina en las escuelas, desde una forma de trabajo que va desde lo concreto hacia lo abstracto. Esta aproximación a la disciplina, según Miranda (2012), aún está presente en el currículum de Geografía, complementada con las modificaciones realizadas en dictadura militar (1973-1990), periodo en el cual, desde una mirada positivista, conductista, y con un objetivo eminentemente nacionalista, se implementó una mirada naturalista de las regiones, donde el énfasis estuvo en la división político administrativa que se instauró en 1974. En este sentido, de acuerdo con Henríquez (2011) y Miranda (2012) los cambios que han sufrido el currículum escolar y, en particular, la enseñanza de la Geografía en Chile, han estado tensionados por sus relaciones con la figura del Estado nación y la conformación de la identidad de los ciudadanos.

Con el ajuste curricular de 2009, los contenidos se estructuran de manera secuencial y no repetitiva. En cuanto a la Geografía, se agrupan en tres dimensiones que progresan durante las etapas escolares: Localización espacial y comprensión sistémica del espacio geográfico; Habilidades de análisis territorial; y Habilidades y actitudes de valoración y actuación responsable con el espacio. De esta forma, de acuerdo con el programa del Ministerio de Educación (Mineduc),

Las habilidades geográficas tienen como propósito desarrollar en alumnos y alumnas la ubicación espacial y el análisis territorial, en el cual han de poder integrar diversas variables para adquirir una visión dinámica y sistémica del espacio que habitan. Las habilidades de ubicación espacial progresan desde primero a quinto básico, en relación a la ubicación y distribución de procesos naturales y sociales. Las habilidades de análisis territorial progresan desde sexto básico hasta cuarto medio, y con ellas se busca que los estudiantes logren integrar variables para explicar la dinámica espacial de distintos procesos en un territorio hasta llegar a una visión sistémica del espacio que habitan, lo que les permitirá comprender que el territorio es una construcción humana y posible de modificar en beneficio de la calidad de vida, contribuyendo de esta manera a la formación de ciudadanos capaces de evaluar las implicancias que tiene el desarrollo sustentable para su vida y la de la humanidad. (2009, p. 197) 
Así entonces, según lo propuesto por el currículum, la Geografía vuelve a incorporarse en todos los niveles, teniendo en su base las discusiones del ajuste curricular en torno a la relevancia del ser humano como eje estructurante de la sociedad y donde se han integrado enfoques teóricos que hoy se han posicionado dentro de la Geografía como disciplina académica.

Sin embargo, esto último ha sido fuertemente criticado por la Sociedad Chilena de Ciencias Geográficas (2013), ya que, a pesar de posicionarse el "pensamiento geográfico" como uno de los énfasis de las Bases Curriculares, se ha omitido su tratamiento o discusión en la secuencia de objetivos que aparecen en los planes y programas de estudio de aprendizajes desde séptimo a segundo medio. Esto llevaría a la asignatura de Historia, Geografía y Ciencias Sociales a privilegiar las reflexiones temáticas en torno al pensamiento temporal, anulando los instrumentos y herramientas conceptuales que se encuentran integrados en el pensamiento espacial, lo que, de acuerdo a la Sociedad Chilena de Ciencias Geográficas, obstaculiza el proceso de aprendizaje, así como las relaciones con otras disciplinas de las Ciencias Sociales y la Formación Ciudadana. De esta forma, a pesar de los énfasis de las Bases Curriculares para la asignatura de Historia, en cuanto a incluir la Geografía dentro de los planes de estudio, existe una estructura cognitiva y de desglose de los conocimientos que no permite el trabajo conjunto de las disciplinas del área.

Para Rodríguez (2010), esta realidad da cuenta de que, en los procesos de aprendizaje, la Geografía ha tendido a asociarse con aprendizajes memorísticos que no tienen repercusión alguna en el desarrollo cognoscitivo de los estudiantes e inclusive de los profesores. En esa línea, los objetivos de aprendizaje propios de la disciplina han sido desplazados hacia habilidades específicas (inclusive solo nombradas en indicadores de evaluación), donde la Geografía funciona como una ciencia auxiliar. El aprendizaje memorístico puede otorgar algunos elementos para que los estudiantes puedan referirse en términos geográficos a la realidad social, sin embargo, si estos elementos no se vuelcan en aprendizajes significativos, se comete el error de no incorporar ni permitir su apropiación crítica, por parte de los estudiantes. Con ello, la progresión propuesta por las Bases Curriculares tiende a desarrollar más aquellos elementos de la Geografía donde la 
concepción del espacio es objetiva y el tipo de aprendizaje se vuelve verbal, procedimental y conceptual.

\section{LA GEOGRAFÍA EN EL CURRÍCULUM ACTUAL}

Las Bases Curriculares, según el Mineduc (2013), es el documento principal del currículum nacional que "cumple la misión de ofrecer una base cultural común para todo el país, mediante Objetivos de Aprendizaje establecidos para cada curso o nivel”. Estas Bases se sustentan en dos grandes cuerpos normados: la Constitución Política y la Declaración Universal de los Derechos Humanos. Ambos cuerpos sostienen una mirada de la educación que se define bajo la libertad de enseñanza y donde el agente que la posibilita es el Estado. De esta forma, las Bases Curriculares deben garantizar que todos los estudiantes del país participen de una experiencia educativa similar, sin embargo, se deja abierta la posibilidad de que esta experiencia sea complementada con otras, a partir de los contextos particulares de las necesidades de los estudiantes y las escuelas. Con estos elementos en consideración, una persona educada bajo las Bases Curriculares posee un perfil integral, reflexivo, situado en la globalidad actual como ciudadano activo y autónomo en sus decisiones.

En particular, en el área de Historia, Geografía y Ciencias Sociales, las Bases Curriculares proponen como ejes centrales para la asignatura: a) La perspectiva multidisciplinar; b) El pensamiento histórico; c) El pensamiento geográfico; d) El Desarrollo de competencias ciudadanas y respeto por los derechos humanos; e) El carácter interpretativo de las Ciencias Sociales; f) La historia de Chile y del mundo bajo una mirada dialógica; g) Las Ciencias Sociales: perspectiva política y económica. La definición de la Geografía como uno de los ejes centrales de la Bases Curriculares ha sido valorada positivamente tanto por los docentes como por los académicos de la disciplina. Sin embargo, estos mismos han advertido la nula presencia de la disciplina en los conocimientos propuestos ya que, al momento de traducir el pensamiento geográfico en aprendizajes esperados, los conocimientos geográficos resultan omitidos, no existiendo mención alguna de ellos. 
En el documento de las Bases Curriculares se señala que "este tipo de pensamiento [geográfico] implica una forma de razonamiento particular, que amplía el ámbito de la experiencia y aporta a los estudiantes una visión integral del mundo que los rodea, desde lo más próximo hasta lo más remoto" (Mineduc, 2013, p.195). Se desprende de esta definición que los estudiantes lograrán tener una visión integral a partir del estudio de lo próximo a lo más lejano, desde una mirada que, si tuviésemos que catalogarla, podríamos considerar piagetiana. Como principios declarados, se señala que el pensamiento geográfico supone comprender el espacio desde la perspectiva de las relaciones dinámicas entre la sociedad y el medio natural, y desde una realidad que es mutable y no ajena al accionar humano. Desde esta mirada, se propone que los estudiantes desarrollen una conciencia fundada respecto del impacto del quehacer en el medio natural. A partir de ello, según el Mineduc, los estudiantes serán capaces de comprender que los fenómenos espaciales son generados de forma multicausal (causas naturales, sociales, económicas, políticas, etc.), por lo que aplicarán y practicarán una conciencia geográfica desde un enfoque sistémico del espacio. Por otra parte, el documento señala que los estudiantes deberán utilizar herramientas geográficas que les permitan representar, caracterizar e identificar diversos patrones de distribución del espacio geográfico. Finalmente, esto se traducirá en el desarrollo de actitudes valóricas por parte de los estudiantes en cuanto a actitudes de cuidado y responsabilidad con el medio en que habitan "comprendiendo que lograr un desarrollo sustentable es un desafío que nos compete a todos (Estado - sociedad - individuos)" (Mineduc, 2013, p.5).

Considerando lo anterior, se constata que las definiciones, al ser traducidas en el diseño curricular, no solo han sufrido deformaciones teórico-pedagógicas, sino que también han sido conscientemente subordinadas a una multiplicidad de indicadores de evaluación, que terminan por anular la relevancia del pensamiento geográfico como uno de los énfasis de la propuesta curricular.

\section{PENSAR GEOGRÁFICAMENTE}


En el momento en que los docentes se enfrentan al currículum escolar, el contexto anteriormente descrito se ve reflejado tanto en el diseño curricular de las clases como en la pérdida o ausencia de herramientas multidisciplinares en la asignatura. En particular, la rutina de exposición y de la transposición didáctica de los contenidos se inclinan hacia el pensamiento temporal-histórico, aspecto que se ve de manera concreta en el plan de estudios de octavo básico, lo que, desde la perspectiva asumida por este estudio, es un problema no solo para los estudiantes como sujetos de educación, sino también para los docentes. Veamos por qué.

El plan de estudios de octavo básico actualizado a 2016, bajo el marco de las Bases Curriculares de 2013, para el primer y segundo semestre tiene como organizadores temáticos:

a) "Los inicios de la modernidad: humanismo, reforma y el choque de dos mundos".

b) "Formación de la sociedad americana y de los principales rasgos del Chile colonial".

c) "Nuevos principios que configuran el mundo occidental: ilustración, revolución e independencia".

d) "Sociedad y territorio: la región en Chile y América" (sobre este último haremos referencia en un apartado particular).

Desde esta perspectiva, las Bases Curriculares (2013) señalan que "En [el plan de estudios de] $8^{\circ}$ básico se ha puesto el acento en los procesos históricos que sientan las bases de la modernidad y que acaban en las revoluciones de los siglos XVIII y XIX” (p.8). Así, en cuanto al pensamiento temporal-histórico y espacial-geográfico, en particular sobre Geografía, se señala que será abordado en el organizador temático centrado en la Región como perspectiva de análisis social y territorial, estudiándose diversos criterios, problemas y aspectos de la realidad regional, tanto de América como de Chile (Mineduc, 2013, p.8). De esta forma, al revisar el programa, la Geografía como disciplina aparece fragmentada en una unidad aislada del programa y donde las herramientas propias de la disciplina aparecen asimiladas a destrezas comunes como la observación, la reflexión y la capacidad de trabajo grupal. Al no ir más allá de la localización en mapas de sitios y lugares "importantes" para el nivel de enseñanza, se dejan de lado habilidades propias de la disciplina como: 
La graficidad, es decir, la capacidad para la representación, el análisis y la interpretación gráfica, abarcando diagramas, redes, y mapas; la visualidad o capacidad de observación, especialmente, a través de la valoración de tamaños y magnitudes y por último el trabajo de campo. (Gallastegui y Rojas, 2016, p.283)

Lo anterior refuerza la noción de aprendizaje que el Mineduc asocia al Pensamiento geográfico, pues:

Se espera que los estudiantes puedan representar, a través del uso de distintas herramientas, tanto la ubicación y características de los lugares, como los diferentes tipos de información geográfica. Junto a esto, se espera que los estudiantes desarrollen la capacidad de interpretar datos e información geográfica a fin de identificar distribuciones espaciales y patrones asociados a las dinámicas del territorio. (Mineduc, 2013, p.10)

Además, existen vacíos como ¿con qué herramientas de la Geografía podrán representar los estudiantes la localización, ubicación y características de los lugares? y ¿qué tipos de información geográfica aprenderán a interpretar los estudiantes? Preguntas que resultan aclaradoras cuando se analiza el programa de octavo básico, pues los primeros tres organizadores temáticos hacen referencia exclusiva al énfasis del pensamiento histórico en la asignatura, esto es, del pensamiento temporal, ya que se pretende que los estudiantes “comprendan que la experiencia de vivir en sociedad está contextualizada en el tiempo, y que en tanto seres humanos, nuestra existencia se desenvuelve en el devenir de la historia" (Mineduc, 2013, p.4). El pensamiento geográfico, por tanto, es arrojado de forma mínima hacia indicadores de evaluación donde el estudiante podrá representar la "ubicación" y características de lugares e información geográfica, e interpretar datos para explicar relaciones (Mineduc, 2016, p.291). Se deja expresar, así, un desconocimiento del espacio, aunque no de la Geografía, como una categoría autónoma del pensar histórico (Santos, 2000).

Frente a esto, las preguntas siguen estando abiertas, por lo que los siguientes tipos de habilidades que se deducen a partir del programa son: 
1. Situar en un contexto espacial, por ejemplo, situando el Humanismo y el Renacimiento en su contexto espacial y temporal; situando los viajes de exploración que trajeron a los europeos a América; situando los procesos revolucionarios de fines del siglo XVIII y comienzos del siglo XIX.

2. Representar y ubicar hitos mediante herramientas geográficas (mapas), por ejemplo, el proceso de conquista del continente americano; los procesos de exploración y conquista; los cambios en las divisiones político-administrativas del continente; las rutas comerciales hacia España; la organización socioespacial de la hacienda, etc.

La materialidad del pensamiento geográfico se traduce, así, en la utilización y construcción de recursos didácticos como los mapas, cartas o gráficos para apoyar los procesos de aprendizaje relativos a los contenidos del curso. Cabe preguntarse ¿es esto suficiente para aprender a pensar geográficamente? Desde la perspectiva asumida en esta investigación, y bajo dos premisas, no. La primera: los recursos de la Geografía, como los mapas, aparecen como elementos en el proceso aprendizaje de los estudiantes de manera descontextualizada, ya que se presentan como recursos que no están presentes en su cotidianidad y que no tienen una utilidad práctica más allá de la actividad en sí misma. La segunda: el currículum propicia un aislamiento de los recursos disciplinares de la Geografía respecto del proceso de enseñanza, por lo que, o se convierten en hitos sin repercusiones, o derechamente se olvidan con el pasar del tiempo, ya que no se ven involucrados en instancias de mayor relevancia pedagógica.

De esta forma, surgen dos preguntas: ¿cuál es la visión de la Geografía que se ha querido instalar a través del Programa? y ¿en qué actitudes de los estudiantes ha de verse reflejada? Por ejemplo: ¿es posible integrar el mundo de las experiencias espaciales o tensionar las representaciones espaciales de los estudiantes con el uso de tales recursos? O como lo plantea Souto (2011), “¿Por qué no se incorporan las preocupaciones cotidianas como objeto de estudio a la Geografía escolar?” (p.29).

Las representaciones clásicas como aquellas que provienen de nuestras experiencias educativas y que quedan expresadas en el siguiente comentario: '...me carga la Geografía, me carga hacer mapas', hacen clara alusión a prácticas pedagógicas que centran su trabajo en localizar hechos en mapas, normalmente asociados a algún 
ejercicio de destreza artístico-motora como pintar sin salirse del margen, y que se refuerzan aún más con frases como ‘...debe quedar parejito', no hacen sino delinear una concepción muy alejada de aquella que realmente han intentado propugnar los geógrafos”. (Sociedad Chilena de Ciencias Geográficas, 2016, p.25)

\section{DEL CURRÍCULUM AL AULA}

Hasta ahora se han analizado las principales orientaciones curriculares respecto de la enseñanza de la Geografía para octavo básico. En este apartado, se expone una evaluación del impacto de estas orientaciones en el proceso de aprendizaje. A continuación, se presentan tres ejes en los que se agrupan los elementos del currículum analizados en los apartados anteriores, con el objetivo de evaluar su impacto en la práctica pedagógica y el proceso de aprendizaje.

\section{a) La fragmentación del trabajo multidisciplinar y la falta de visión de totalidad}

El trabajo multidisciplinar es uno de los mayores desafíos en la escuela. Un ejemplo de problemáticas asociadas a este eje es el predominio de la visión temporal-histórica en la problematización de los contenidos, así como una forma fragmentaria y secuencial de aprendizaje que no permite la apropiación de los conocimientos por parte de los estudiantes, por lo que se recurre a métodos de memorización y repetición que generan vacíos de contenido y hacen perder el sentido de asistir a la escuela. En el caso de octavo básico, los estudiantes recuerdan, de lo visto en séptimo básico sobre las civilizaciones inca y azteca, elementos del desarrollo político-religioso y de las caídas demográficas tras la llegada de los españoles, mas no de la ciencia y del desarrollo científico expresado en el conocimiento de la naturaleza, el trabajo de la tierra y la concepción del espacio (pensamiento espacial).

Una de las grandes ignorancias que heredamos del mundo europeo dice relación con la gran riqueza que emanaba de las técnicas agrícolas y mineras, así como de las normas 
para la conservación y protección ecológicas que venían desarrollando los pueblos originarios de América. Algunas de estas técnicas perduran hasta hoy e incluso se han vuelto masivas, como es el caso de la producción hidropónica de origen azteca y las instancias de reflexión sobre estas experiencias, desde la perspectiva del pensar geográfico, podrían contribuir al respeto y defensa del medio ambiente frente a la producción industrial nociva.

\section{b) La imprecisión de las habilidades geográficas a desarrollar y, por consiguiente, la indefinición de las herramientas propias de la Geografía a utilizar}

Si bien, para el nivel de octavo básico, el currículum declara que se desarrollarán de forma progresiva las habilidades de análisis territorial, con la finalidad de que los estudiantes logren integrar diversas variables en la comprensión de la dinámica espacial de los territorios, bajo un enfoque sistémico del espacio, no existe ningún aprendizaje esperado que dé cuenta de las habilidades específicas del nivel, así como de las herramientas que ayudarán a cumplir tales aprendizajes.

En el plan de estudio se espera que los estudiantes puedan representar en mapas, por ejemplo, los principales hitos de las rutas e itinerarios de las empresas de conquista en América así como su justificación. El indicador de evaluación -que bajo las Bases Curriculares se traduce en habilidades específicas- para este objetivo será: "Representar mediante herramientas geográficas el proceso de conquista del continente americano, identificando la lógica seguida por los conquistadores para ocupar el territorio". La herramienta geográfica a utilizar es el mapa, sin embargo, las representaciones iniciales de los estudiantes en torno a la construcción de un mapa se inclinan hacia su carácter estético, en tanto es necesario calcar la silueta del país y situar los puntos tal cual la imagen, pero no como un recurso de información. Es decir, más allá de representar el proceso de conquista, la actividad se convierte en dibujar el título, la leyenda, la escala, la brújula con puntos cardinales y las coordenadas geográficas, todo lo cual se transforma automáticamente en aspectos incomprensibles para los estudiantes, no por el hecho de que no puedan entender qué significa cada uno de estos elementos, sino porque el trabajo con mapas no está integrado como un material didáctico o recurso permanente en la asignatura de historia. Los 
mapas, además de situar lugares, no se utilizan para el análisis, por tanto, son incorporados de manera auxiliar y esporádica.

\section{c) La ausencia del pensar geográfico en el programa de estudio}

Este punto ha sido abordado a lo largo del artículo, sin embargo, merece atención el efecto que genera la falta de rigurosidad en el desarrollo del pensamiento geográfico en el currículum. La Sociedad Chilena de Ciencias Geográficas ha desarrollado una propuesta educativa en torno a la enseñanza de la Geografía en Chile, donde ha incluido como necesidades urgentes la incorporación de temáticas actuales como los patrones espaciales de ocupación humana, la interrelación entre la sociedad y la naturaleza, la concepción holística del planeta como hogar del ser humano, los impactos espaciales del proceso de globalización, el calentamiento global, los flujos migratorios, la urbanización acelerada, la localización de empresas transnacionales, la relación de Chile y sus regiones con la economía global, y los tratados internaciones y sus impactos en el espacio geográfico. Asimismo, esta perspectiva de problematización debiese estar permeada por los aspectos históricos, económicos y políticos.

Cabe señalar, finalmente, que estos tres grandes nudos confunden las trayectorias de aprendizajes formales e informales (Gallastegui y Rojas, 2016) de las y los estudiantes, ignorando, de esta manera, los siguientes aspectos fundamentales:

\section{c.1. EI mundo de las experiencias espaciales}

Como un primer acercamiento hacia una conceptualización de los elementos del conocimiento geográfico, las experiencias espaciales propias de los estudiantes debieran contribuir a la percepción espacial, así como a la localización y ubicación. Sin embargo, no son consideradas, ya que se parte desde supuestos y definiciones dadas que, sobre todo, tienden a la homogeneización de las representaciones que tienen los estudiantes.

\section{c.2. La esfera de las representaciones espaciales}


A partir de esto último, el currículum, en términos del pensamiento geográfico, utiliza sin distinciones conceptos como paisaje, medio natural, territorio, espacio geográfico o configuración territorial. Esto hace que la transposición didáctica de los contenidos genere problemáticas al docente, quien debe lidiar con una conceptualización confusa y que, por tanto, le presenta dificultades a la hora de organizar esquemáticamente los contenidos, lo que, a su vez, genera un desorden en las representaciones que ya tenían los estudiantes. Esto redunda en que, finalmente, no se logre un aprendizaje significativo. Como han planteado Gallastegui y Rojas (2016, p.23), “el conocimiento cotidiano resignifica de modo informal parte del 'saber enseñado' y parte del 'saber sabio' y lo utiliza para interpretar el presente en clave de actualidad", por lo que las representaciones espaciales tenderán a confundirse conceptualmente.

\section{c.3. El campo del conocimiento}

En tercer y último lugar, el campo del conocimiento pareciera ser ignorado por las Bases Curriculares y mucho más aún por los planes de estudios, ya que las herramientas disciplinares como los sistemas de información geográfica han sido omitidos en su traducción a habilidades. Estos, a pesar de corresponder a un nivel cognoscitivo de mayor complejidad, en tanto su desarrollo depende de los debates y encuentros en el ámbito de la Geografía académica, debiesen permear el conocimiento geográfico escolar que se ha quedado estancado en teorizaciones y discusiones de comienzos del siglo XX.

\section{CONCLUSIONES}

A lo largo de este artículo se analizó y evaluó la concepción de la Geografía como disciplina en el currículum y sus expresiones en el diseño de los planes de estudio. En primer lugar, se señaló que los objetivos de aprendizaje propios de la disciplina geográfica han sido desplazados hacia habilidades específicas, por lo que el trabajo multidisciplinar en la asignatura de Historia, Geografía y Ciencias Sociales sigue siendo una deuda pendiente, en la medida en que pareciera haber un desconocimiento del espacio como una categoría autónoma del pensar histórico. En segundo lugar, el estado del currículum ha generado 
problemáticas en el tratamiento pedagógico de los contenidos como ausencias en los procesos de aprendizaje de los estudiantes, lo que ha tenido repercusiones tanto en la percepción cognitiva de las temáticas, como en la fragmentación del conocimiento.

Por otro lado, las posibilidades de los docentes de innovar frente al currículum han sido limitadas de manera arbitraria, por lo que, finalmente, lo evaluable, en función del cumplimiento de los planes de estudio, son las orientaciones curriculares dispuestas en los documentos del Mineduc. Así, en tercer lugar, tanto la indefinición de habilidades propias geográficas así como de las herramientas disciplinares, genera la reproducción de un modelo pedagógico insuficiente para promover el pensamiento geográfico entre los estudiantes de octavo básico y depende de una decisión ético-profesional de los docentes transformar este modelo o no. Todo esto tiene como consecuencia la incomprensión y descontextualización de fenómenos globales y locales, sobre todo porque las memorias colectivas no han sido incorporadas aún como recursos de conocimiento.

En síntesis, a partir de los principales resultados expuestos, es posible señalar que existe una incongruencia entre el código curricular y el currículo. Ello se debe a que, a pesar de que las Bases Curriculares declaran la necesidad de una perspectiva de enseñanza y aprendizaje multidisciplinar para el área de Historia, Geografía y Ciencias Sociales, en el currículo no existe una red de objetivos de aprendizaje e indicadores de evaluación que permitan integrar de forma significativa a la disciplina geográfica, en tanto desarrollo del pensamiento geográfico en los estudiantes. De esta forma, la Geografía escolar continúa siendo una ciencia anecdótica, auxiliar y estancada en las discusiones académicas del siglo XX.

\section{REFERENCIAS}

Carr, D. (2005). El sentido de la educación: una introducción a la filosofía y a la teoría de la educación y de la enseñanza. Barcelona, España: Graó.

Grundy, S. (1998). Producto o praxis del currículum. Madrid, España: Morata. 
Gallastegui, J. y Rojas, I. (2016). Aprehender el espacio geográfico: del conocimiento intuitivo al concientizado. Valparaíso, Chile: Universidad de Playa Ancha (Convenio de Desempeño).

Henríquez, R. (2011). Un balance provisional de la investigación en enseñanza y aprendizaje de la historia en Chile en los últimos 30 años. Clio\&Asociados. La Historia Enseñada, 15. 9 - 26.

Lefebvre, H. (1974). La producción del espacio. Madrid, España: Capitán Swing.

Lundgren, U. (1992). Teoría del currículum y escolarización. Madrid, España: Ediciones Morata.

Magendzo, A. (2008). Dilemas del currículum y la pedagogía. Santiago, Chile: LOM Ediciones.

Ministerio de Educación. (2009). Actualización Curricular 2009. Santiago: Formación General.

Ministerio de Educación. (2013). Bases Curriculares $7^{\circ}$ a $2^{\circ}$ medio. Santiago, Chile: Unidad de Currículum y Evaluación.

Ministerio de Educación. (2016). Programa de Estudio Octavo Básico. Santiago, Chile: Unidad de Currículum y Evaluación.

Miranda, P. (2012). La educación geográfica en Chile: Desde su aparición en el currículum escolar en el siglo XIX hasta los ajustes curriculares de 2010. Revista Virtual ANEKUMENE Geografia, Cultura y Educació (4), 51-71.

Núñez, I. (1997). Historia reciente de la educación chilena. Recuperado de: http://www.oei.es/historico/quipu/chile/CHIL02.PDF 
Pinto, R. (2008). El currículum crítico. Una pedagogía transformativa para la educación latinoamericana. Santiago: Ediciones Universidad Católica de Chile.

Rodríguez, E. (2010). Geografía conceptual: Enseñanza y aprendizaje de la Geografía en la Educación Básica secundaria. Bogotá, Colombia: Tercer Mundo.

Santos, M. (2000). La naturaleza del espacio. Técnica y tiempo. Razón y emoción. Barcelona: Ariel Geografía.

Sociedad Chilena de Ciencias Geográficas (2013). Informe de Revisión Bases Curriculares $7^{\circ}$ Básica a $2^{\circ}$ Medio. Santiago, Chile: Universidad Alberto Hurtado.

Sociedad Chilena de Ciencias Geográficas (2016). Una educación geográfica para Chile. Santiago, Chile: Comisión de Educación Geográfica de la Sociedad Chilena de Ciencias Geográficas y Universidades Chilenas.

Souto, X. (2011). Una educación geográfica para el siglo XXI: Aprender competencias para ser ciudadano en el mundo global. Revista Virtual ANEKUMENE Geografía, Cultura y Educación, (1), 28-47. 